PROCEEDINGS OF THE

AMERICAN MATHEMATICAL SOCIETY

Volume 125, Number 11, November 1997, Pages 3255-3256

S 0002-9939(97)03906-3

\title{
THE SCHWARZ-PICK LEMMA FOR DERIVATIVES
}

\author{
A. F. BEARDON
}

(Communicated by Theodore W. Gamelin)

\begin{abstract}
The Schwarz-Pick Lemma states that any analytic function of the unit disc into itself is a contraction with respect to the hyperbolic metric. In this note a related result is proved for the derivative of an analytic function.
\end{abstract}

\section{INTRODUCTION}

In [2] Dieudonne showed that if $f: \mathbb{D} \rightarrow \mathbb{D}$ is analytic, where $\mathbb{D}$ is the open unit disc in the complex plane, and if $f(0)=0$, then

$$
\left|f^{\prime}(z)\right| \leq \begin{cases}1 & \text { if }|z| \leq \sqrt{2}-1 \\ \frac{\left(1+|z|^{2}\right)^{2}}{4|z|\left(1-|z|^{2}\right)} & \text { if }|z| \geq \sqrt{2}-1 .\end{cases}
$$

He then suggested (in the footnote on page 352 ) that this inequality, which is best possible for each value of $|z|$, should be considered as a Schwarz Lemma for the derivative $f^{\prime}$. In this note we derive a more elegant version of this that is closer to the classical Schwarz-Pick Lemma.

As $f$ maps $\mathbb{D}$ into itself one can argue that any discussion of $f$ should be in terms of the hyperbolic metric rather than the Euclidean metric. The disk $\mathbb{D}$ is endowed with the hyperbolic metric $\rho$ derived from $2|d z| /\left(1-|z|^{2}\right)$, and the hyperbolic derivative $f^{*}(z)$ of $f$ at $z$ is given by

$$
f^{*}(z)=\left(\frac{1-|z|^{2}}{1-|f(z)|^{2}}\right) f^{\prime}(z) .
$$

Pick's version of Schwarz's Lemma, namely that $\rho(f z, f w) \leq \rho(z, w)$, guarantees that $\left|f^{*}(z)\right|<1$ in $\mathbb{D}$ (unless $f$ is a Möbius map of $\mathbb{D}$ onto itself) and this means that we can measure the hyperbolic distance between two hyperbolic derivatives. This leads to the following Schwarz-Pick Lemma for derivatives.

Theorem. If $f: \mathbb{D} \rightarrow \mathbb{D}$ is analytic but not a conformal automorphism of $\mathbb{D}$, and if $f(0)=0$, then

$$
\rho\left(f^{*}(0), f^{*}(z)\right) \leq 2 \rho(0, z) .
$$

Further, equality holds in (2) for each $z$ when $f(z)=z^{2}$.

Received by the editors May 1, 1996.

1991 Mathematics Subject Classification. Primary 30F45; Secondary 30C80.

Key words and phrases. Analytic, Schwarz-Pick, hyperbolic.

(C)1997 American Mathematical Society 
If $f(z)=z^{2}$, then $f^{*}(0)=0$ and $f^{*}(z)=2 z /\left(1+|z|^{2}\right)$, and a simple calculation using the fact that

$$
\rho(0, z)=\log \frac{1+|z|}{1-|z|}
$$

shows that equality holds in (2).

\section{The Proof of the Theorem}

We begin with a preliminary result.

Lemma 1. Let $z_{0}$ and $w_{0}$ be points of $D$ with $\left|w_{0}\right|<\left|z_{0}\right|$. If $f: \mathbb{D} \rightarrow \mathbb{D}$ is analytic, and if $f(0)=0$ and $f\left(z_{0}\right)=w_{0}$, then both $f^{*}(0)$ and $f^{*}\left(z_{0}\right)$ lie in the closed hyperbolic disc

$$
D=\left\{z: \rho\left(z, w_{0} / z_{0}\right) \leq \rho\left(0, z_{0}\right)\right\} .
$$

Proof. Given $z_{0}$ and $w_{0}$, we define maps $h: \mathbb{D} \rightarrow \mathbb{D}$ and $g: \mathbb{D} \rightarrow \mathbb{D}$ by

$$
h(z)=\frac{f(z)}{z}, \quad \frac{f(z)-f\left(z_{0}\right)}{1-f(z) \overline{f\left(z_{0}\right)}}=g(z)\left(\frac{z-z_{0}}{1-z \overline{z_{0}}}\right) .
$$

It is immediate that

$$
h(0)=f^{\prime}(0)=f^{*}(0), \quad h\left(z_{0}\right)=\frac{w_{0}}{z_{0}}, \quad g(0)=\frac{w_{0}}{z_{0}}, \quad g\left(z_{0}\right)=f^{*}\left(z_{0}\right),
$$

so that, by applying Pick's Lemma to $h$ and $g$,

$$
\rho\left(f^{*}(0), w_{0} / z_{0}\right) \leq \rho\left(0, z_{0}\right), \quad \rho\left(f^{*}\left(z_{0}\right), w_{0} / z_{0}\right) \leq \rho\left(0, z_{0}\right) .
$$

This shows that $f^{*}(0)$ and $f^{*}(z)$ lie in the closed disc $D$.

The inequality (2) (with $z$ replaced by $z_{0}$ ) is an immediate consequence of (4) and the Triangle inequality, and this completes the proof of the Theorem. For a Euclidean version of Lemma 1, see [1], p. 19 and [3], p. 198.

We end with the remark that if equality holds in $(2)$, then $f^{*}(0)$ and $f^{*}(z)$ must lie at diametrically opposite points on the boundary of $D$, so that equality holds in both of the inequalities in (4). We deduce that $h$ is a Möbius map of $\mathbb{D}$ onto itself and hence $f(z)=z h(z)$, a Blaschke product of degree two.

\section{REFERENCES}

1. C. Caratheodory, Theory of functions of a complex variable, Vol. II, Chelsea, 1960. MR 16:346c

2. J. Dieudonne, Recherches sur quelques problemes relatifs aux polynomes et aux fonctions bornees d'une variable complexe, Ann. Sci. Ecole Norm. Sup. 48 (1931), 247-358.

3. P. L. Duren, Univalent functions, Springer-Verlag, 1983. MR 85j:30034

Department of Pure Mathematics \& Mathematical Statistics, University of CamBridge, 16 Mill Lane, Cambridge, CB2 1SB, England

E-mail address: A.F.Beardon@dpmms.cam.ac.uk 